\title{
Nonequilibrium statistical operators for systems with finite lifetime
}

\author{
V.V. Ryazanov \\ Institute for Nuclear Research of the National Academy of Sciences of Ukraine \\ 47 Nauky Prosp., Kiev 03068, Ukraine \\ E-mail: vryazan@kinr.kiev.ua
}

Received January 18, 2007

\begin{abstract}
A family of nonequilibrium statistical operators (NSO) is introduced which differ by the system lifetime distribution over which the quasiequilibrium (relevant) distribution is averaged. This changes the form of the source in the Liouville equation, as well as the expressions for the kinetic coefficients, average fluxes, and kinetic equations obtained with use of NSO. The difference from the Zubarev form of NSO is of the order of the reciprocal lifetime of a system.
\end{abstract}

PACS: 02.50.-r Probability theory, stochastic processes, and statistics;

05.70.Ln Nonequilibrium and irreversible thermodynamics;

31.70.Hq Time-dependent phenomena: excitation and relaxation processes, and reaction rates.

Keywords: nonequilibrium statistical operator, lifetime distributions, past, present.

In work [1] the new interpretation of a method of the Nonequilibrium Statistical Operator (NSO) [2,3] is given, in which NSO is treated as averaging of the quasi-equilibrium (or relevant $[4,5]$ ) statistical operator on the system past lifetime distribution and NSO rewritten as

$$
\begin{gathered}
\ln \rho(t)=\int_{0}^{\infty} p_{q}(u) \ln \rho_{q}(t-u,-u) d u, \\
\ln \rho_{q}(t, 0)=-\Phi(t)-\sum_{n} F_{n}(t) P_{n} ; \\
\ln \rho_{q}\left(t, t_{1}\right)=\exp \left\{-t_{1} H / i \hbar\right\} \ln \rho_{q}(t, 0) \exp \left\{t_{1} H / i \hbar\right\} ; \\
\Phi(t)=\ln \operatorname{Sp} \exp \left\{-\sum_{n} F_{n}(t) P_{n}\right\},
\end{gathered}
$$

where $H$ is hamiltonian, $\ln \rho(t)$ is the logarithm of the $\mathrm{NSO}, \ln \rho_{q}(t, 0)$ is the logarithm of the quasi-equilibrium distribution; the first time argument indicates the time dependence of the values of the thermodynamic parameters $F_{m}$; the second time argument $t_{2}$ in $\rho_{q}\left(t_{1}, t_{2}\right)$ denotes the time dependence through the Heizenberg representation for dynamical variables $P_{m}$ from which $\rho_{q}(t, 0)$ can depend [1-3]. In [1] the function $p_{q}(u)=\varepsilon \exp \{-\varepsilon u\}$ from $[2,3]$ was interpreted as the probability distribution density of the lifetime of a system from the random moment $t_{0}$ of its birth till the current moment $t ; u=t-t_{0}$. This time period can be called the time period of getting information about system from its past. Instead of the exponential distribution $p_{q}(u)$ in (1) any other sample distribution could be taken. The arbitrary kind of lifetime density distribution $p_{q}(u)$ enables to write down a general view of a source in the dynamic Liouville equation, which thus accepts Boltzmann-Prigogine form and contains dissipative effects [4,5]. It is known [2,3] that the Liouville equation for Zubarev's NSO contains the source $J=J_{\text {zub }}=$ $=-\varepsilon\left[\ln \rho(t)-\ln \rho_{q}(t, 0)\right]$ which tends to zero after taking the thermodynamic limit and setting $\varepsilon \rightarrow 0, \varepsilon=<t-t_{0}>^{-1}$, which in the spirit of the paper [1] corresponds to the infinitely large lifetime value of an infinitely large system. For a system with finite size this source is not equal to zero. Besides the Zubarev's form of NSO [2,3], Green-Mori form [6,7] is known, where one assumes the auxiliary weight function [5] to be equal $W\left(t, t^{\prime}\right)=1-$ $-\left(t-t^{\prime}\right) / \tau ; \mathrm{w}\left(t, t^{\prime}\right)=d W\left(t, t^{\prime}\right) / d t^{\prime}=1 / \tau ; \tau=t-t_{0}$. After averaging one sets $\tau \rightarrow \infty$. This situation at $p_{q}\left(u=t-t_{0}\right)=$ $w\left(t, t^{\prime}=t_{0}\right)$ coincides with the uniform lifetime distribution. The source in the Liouville equation takes the form $J=\ln \rho_{q} / \tau$. In [2] this form of NSO is compared to the Zubarev's form.

One could name many examples of explicit defining of the function $p_{q}(u)$ in (1). Every definition implies some specific form of the source term $J$ in the Liouville equation, some specific form of the modified Liouville opera- 
tor and NSO. Thus the family of NSO is defined. In [8] it was shown that the lifetime $t-t_{0}$ distribution, the «age» of a system, at big $t^{\text {s }}$ s tends to the exponential form. In NSO $[2,3]$ the influence of the past on the current moment is taken into account. The lifetime distribution need not be exponential. For example, one may choose the $p_{q}(u)$ function in (1) as

$$
p_{q}(u)=\varepsilon^{2} u \exp \{-\varepsilon u\}, u=t-t_{0},
$$

that is in the form of gamma-distribution $p_{q}(u)=$ $=\varepsilon(\varepsilon u)^{k-1} \exp \{-\varepsilon u\} / \Gamma(k),(\Gamma(k)$ is gamma - function $)$ at $k=2$. In this case the distribution (2) coincides with the special Erlang distribution of the order 2 [9]. The logarithm of NSO in this case has the form

$$
\begin{gathered}
\ln \rho(t)=\int_{0}^{\infty} p_{q}(u) \ln \rho_{q}(t-u,-u) d u= \\
=\int_{0}^{\infty} \varepsilon^{2} u \exp \{-\varepsilon u\} \ln \rho_{q}(t-u,-u) d u= \\
=\ln \rho_{q}(t, 0)+\int_{0}^{\infty} \sigma(t-u,-u)(1+\varepsilon u) \exp \{-\varepsilon u\} d u= \\
=\ln \rho_{\mathrm{zub}}(t)+\int_{0}^{\infty} \sigma(t-u,-u) \varepsilon u \exp \{-\varepsilon u\} d u ; \\
\sigma(t-u,-u)=d \ln \rho_{q}(t-u,-u) / d u,
\end{gathered}
$$

where

$$
\begin{aligned}
\ln \rho_{\mathrm{zub}}(t) & =\ln \rho_{q}(t, 0)+\int_{0}^{\infty} \sigma(t-u,-u) \exp \{-\varepsilon u\} d u= \\
& =\int_{0}^{\infty} \ln \rho_{q}(t-u,-u) \varepsilon \exp \{-\varepsilon u\} d u
\end{aligned}
$$

is the Zubarev's form of the NSO,

$$
\sigma(t)=\partial \ln \rho_{q}(t-u,-u) / \partial u_{\mid u=0}=-\partial \ln \rho_{q}(t, 0) / \partial t
$$

is the entropy production operator [2]. The source in the rhs of the Liouville equation equal $J=-\varepsilon[\ln \rho(t)-$ $\left.-\ln \rho_{\mathrm{zub}}(t)\right]$, that is the system relaxes not towards $\ln \rho_{q}(t, 0)$, like it is the case of Zubarev's NSO, but towards $\ln \rho_{\mathrm{zub}}(t)$

The physical results obtained with use of correlation (3) contains additional terms in comparison to Zubarev's NSO. The additional terms describe the influence of the lifetime finiteness on the kinetic processes. The expressions for average fluxes [2] averaged over (3) have the form

$$
\begin{gathered}
<j^{m}(x)>=<j^{m}(x)>_{\mathrm{zub}}+\sum_{n} \iint_{-\infty}^{t} \varepsilon\left(t-t^{\prime}\right) \times \\
\times \exp \left\{\varepsilon\left(t^{\prime}-t\right)\right\}\left(j^{m}(x), j^{n}\left(x^{\prime}, t^{\prime}-t\right)\right) X_{m}\left(x^{\prime}, t^{\prime}\right) d t^{\prime} d x^{\prime},
\end{gathered}
$$

where

$$
\begin{gathered}
<j^{m}(x)>_{\mathrm{zub}}=<j^{m}(x)>_{l}+ \\
+\sum_{n} \iint_{-\infty}^{t} \exp \left\{\varepsilon\left(t^{\prime}-t\right)\right\}\left(j^{m}(x), j^{n}\left(x^{\prime}, t^{\prime}-t\right)\right) X_{m}\left(x^{\prime}, t^{\prime}\right) d t^{\prime} d x^{\prime}
\end{gathered}
$$

are fluxes in the form obtained by Zubarev [2], $j^{n}$ are flux operators, $X_{m}$ are corresponding thermodynamical forces;

$$
\begin{gathered}
\left(j^{m}(x), j^{n}\left(x^{\prime}, t\right)\right)= \\
=\beta^{-1} \int_{0}^{\beta}<j^{m}(x)\left(j^{n}\left(x^{\prime}, t, i \tau\right)-<j^{n}\left(x^{\prime}, t\right)>>>{ }_{l} d \tau\right.
\end{gathered}
$$

are quantum time correlation functions,

$$
\begin{aligned}
j^{n}\left(x^{\prime}, t, \mathrm{i} \tau\right) & =\exp \left\{-\beta^{-1} A \tau\right\} j^{n}\left(x^{\prime}, t\right) \exp \left\{\beta^{-1} A \tau\right\} ; \\
A & =\sum_{m} \int F_{m}(x, t) P_{m}(x) d x .
\end{aligned}
$$

The collision integrals of the generalized kinetic equation [2] averaged over (3) have the amendments also. The same is valid for the generalized transport equations [2], kinetic coefficients etc. Thus the selfdiffusion coefficient (or, to be exact, its Laplace transform over time and space) obtained in [3] in the form

$$
\left.D(\omega, q)=q^{-2} \Phi(\omega, q) / 1+\Phi(\omega, q) /(i \omega-\varepsilon)\right],
$$

where

$$
\begin{gathered}
\Phi(\omega, q)= \\
=\int_{0}^{\infty} d t \exp \{(i \omega-\varepsilon) t\} \int_{0}^{\beta}\left\langle\dot{n}_{q} \dot{n}_{-q}(-t+i \hbar \lambda) d \lambda\right\rangle / \int_{0}^{\beta}\left\langle n_{q} n_{-q}(i \hbar \lambda)\right\rangle d \lambda, \\
n_{q}=\int n(x) \exp \{i(q x)\}=\sum_{j} \exp \left\{i\left(q x_{j}\right)\right\}, \\
n(x)=\sum_{j} \delta\left(x-x_{j}\right),
\end{gathered}
$$

after use of (3) takes on the form

$$
\begin{gathered}
D(\omega, q)= \\
q^{-2}[\Phi(\omega, q)+\varepsilon d \Phi(\omega, q) / d(i \omega)] /\{1+\Phi(\omega, q) /(i \omega-\varepsilon)+ \\
+\varepsilon[d \Phi(\omega, q) / d(i \omega)-\Phi(\omega, q) /(i \omega-\varepsilon)] /(i \omega-\varepsilon)\} .
\end{gathered}
$$

At $\varepsilon \rightarrow 0$, for infinitely large system in the thermodynamic limit this expression (6) coincides with (5) at $\varepsilon \rightarrow 0$ [3]. For finite size systems (as well as for the case $\omega \rightarrow 0$ ) the results differ.

If one chooses as $p_{q}(u)$ in (1) the special Erlang distribution with $k=2,3,4, \ldots, n$ and $P_{q}(x)=1-\exp \{-\varepsilon x\} \times$ $\times\left[1+\varepsilon x / 1 !+\ldots+(\varepsilon x)^{k-1} /(k-1) !\right] ; d P_{q}(x) / d x=p_{q}(x) ; \varepsilon=$ $=k /\langle\Gamma\rangle$, which depends only on the average value of $<\Gamma=u=t-t_{0}>$, that is on the age of the system, in our case (if $k=1$ the result coincides with the exponential dis- 
tribution) at $k=n$ we obtained that the amendment to $\ln$ $\rho_{\mathrm{zub}}(t)$ contains $n-1$ terms, the iteration procedure is performed. As it is indicated in [9] in this case the failure occurs after $k$ stages, and the durations of those stages are independent random values distributed exponentially. Thus the multistage model of the system past is introduced. Nonequilibrium processes typically proceed on different stages, each characterized by a proper time scale [10]. In the distribution (2) the account for two stages is performed. Other distributions may account for some other peculiarities of the system past. The corresponding amendments will enter the expressions for the fluxes, collision integral and kinetic coefficients. The expression for the source in the rhs of the Liouville equation has the form $J=-\varepsilon\left[\ln \rho_{n, \varepsilon}(t)-\ln \rho_{n-1, \varepsilon}(t)\right]$, that is $n$-distribution relaxes towards $(n-1)$-distribution. In general case any functions $p_{q}(u)$ the source is:

$J=p_{q}(0) \ln \rho_{q}(t, 0)+\int_{0}^{\infty}\left(\partial p_{q}(u) / \partial u\right)\left(\ln \rho_{q}(t-u,-u)\right) d u$.

The use of some other explicit forms of the lifetime distributions as $p_{q}(u)$ in (1) (namely, logarithmic logistic distribution with $p_{q}(u)=k \rho^{k} u^{k-1} /\left[1+(u \rho)^{k}\right]^{2}$, complex exponential distribution, obtained if the parameter of the intensity of the exponential distribution itself is a random value [11] which gives the Pareto distribution $p_{q}(u)=$ $=k\left(k / \rho_{0}\right)^{k} /\left(u+k / \rho_{0}\right)^{k+1}$, and so on) makes us to state that the deviation of the distribution of $\ln \rho(t)$ obtained with use of those distributions from $\ln \rho_{\mathrm{zub}}(t)$ is of the order $1 /<\Gamma>$, as in (3), (4), (6).

Thus in big systems their state in the current time moment is influenced only by the existence of the past of the system, its duration, that is the age of a system, and the peculiarities of the system history have only minor influence. The distribution of a form $\varepsilon^{2} u \exp \{-\varepsilon u\}$ correctly describes behaviour of system at small times, existence of some minimal lifetime, as in the case (2) the system has low probability to die at small values of $u$, contrarily to the exponential distribution where this probability is maximal. Any system exist during at least some minimal time.

During evolution the system passes various stages (kinetic, hydrodynamic, etc [10]). Lifetime can end at any stage. At different stages the functions $\rho_{q}$ accept a various kind. Therefore and expression for NSO (1) becomes complicated:

$$
\begin{gathered}
\ln \rho(t)=\int_{0}^{\infty} p_{q}(u) \Sigma_{q}(u) d u, \\
\Sigma_{q}(u)=\sum_{i=0}^{n} \ln \rho_{q i}(t-u,-u) \chi_{\Delta t i} \chi_{\Gamma} ;
\end{gathered}
$$

$$
\begin{gathered}
\chi_{\Delta t i}=1, u \in \Delta t_{i}, \quad \chi_{\Delta t i}=0, \quad u \notin \Delta t_{i} ; \quad \chi_{\Gamma}=1, \\
\sum_{j=1}^{i} \Delta t_{j} \leq \Gamma, \chi_{\Gamma}=0, \sum_{j=1}^{i} \Delta t_{j}>\Gamma ; \\
\Delta t_{0}=t_{\text {chaos }}-t_{0} ; \quad \Delta t_{1}=t_{\text {kin }}-t_{\text {chaos }} ; \\
\Delta t_{2}=t_{\text {gydr }}-t_{\text {kin }} ; \quad \Delta t_{3}=t_{\mathrm{eq}}-t_{\text {gydr }} ; \quad \Delta t_{4}=t_{f}-t_{\mathrm{eq}},
\end{gathered}
$$

where $t_{\text {chaos }}$ is the end moment of the chaotic stage, $t_{f}$ is random moment of system death etc. Generally a problem is complex: it is necessary to know values $\Delta t_{j}, \Gamma, \ln \rho_{q i}$, $p_{q}(u)$. For specification of the description it is possibly to consider various functions $p_{q i}(u)$ for different $\Delta t_{i}$.

The choice of distribution of the lifetime in NSO is connected in view of influence of the past of system, his physical features, on the present moment, for example, to the account only of age of system, as in Zubarev NSO, or with more detailed characteristic of past evolution of the system. The additives to Zubarev results are of the order of the reciprocal past lifetime of a system and are essential at early stages of evolution of system and to systems with small lifetime, where one should not neglect the value $\left\langle\Gamma>^{-1}\right.$. Chosen of Zubarev the form of distribution for the lifetime represents limiting distribution.

1. V.V. Ryazanov, Fortschritte der Physik/Progress of Physics 49, 885 (2001).

2. D.N. Zubarev, Nonequilibrium Statistical Thermodynamics, Plenum-Consultants Bureau, New York (1974).

3. D.N. Zubarev, in: Rev. Science Techn.: Modern Probl. Math., R.B. Gamkrelidze (ed.), Nauka, Moscow (1980), v. 15 p. 131 [J. Soviet Math. 16, 1509 (1981)].

4. A.R. Vasconcellos, R. Luzzi, and L.S. Garcia-Colin, Phys. Rev. A43, 6622 (1991); Phys. Rev. A43, 6663 (1991).

5. J.G. Ramos, A.R. Vasconcellos, and R. Luzzi, Fortschritte der Physik/Progress of Physics 43, 265 (1995).

6. M.S. Green, J. Chem. Phys. 20, 1281 (1952); ibid. 22, 398 (1954).

7. H. Mori, I. Oppenheim, and J. Ross, in: Studies in Statistical Mechanics I, J.de Boer and G.E. Uhlenbeck (eds.), North-Holland, Amsterdam (1962), p. 217.

8. W. Feller, An Introduction to Probability Theory and its Applications, J. Wiley (ed.), New York (1971), v. 2.

9. D.R. Cox, Renewal Theory, J. Wiley (ed.), London, Methuen, New York (1961).

10. N.N. Bogoliubov, in: Studies in Statistical Mechanics, I. J. de Boer and G.E. Uhlenbeck (eds.), North Holland, Amsterdam (1962), p. 4.

11. D.R. Cox and D. Oakes, Analysis of Survival Data, Chapman and Hall, London (1984). 